\title{
Lepadarg
}

III

RÉFLEXIONS HISTORICO-RELIGIEUSES AUTOUR DE SARAPIS HISTORICAL-RELIGIOUS QUESTIONS ABOUT SERAPIS (Reflexões histórico-religiosas em torno de Serápis) 


\title{
Réflexions historico-religieuses autour de Sarapis
}

\author{
Ennio Sanzi ${ }^{1}$ \\ Hugoni Bianchi \\ et loanni Carolo Montesi \\ magistris carissimis \\ sacrum
}

\begin{abstract}
Résumé: Cette étude, ayant pour point de départ un recueil des données prosopographiques du culte d'Isis, s'est transformée en une recherche historico-religieuse sur la réalité religieuse impliquée dans la croyance et dans la vénération des dieux égyptiens Sarapis et Isis en contexte romain. Nous analysons, en particulier, les indices et les manifestations de ces croyances dans la ville de Rome, où ces cultes ont atteint leur plein développement au cours du Ille siècle après Jésus-Christ. L'étude comprend des interprétations sur le profil des adeptes de ces divinités, mettant en évidence la participation des affranchis, des esclaves et surtout des femmes, au-delà de la dimension ethnique, qui a été également analysée. Les pratiques cultuelles, les attentes et les concepts liés à ces deux divinités sont nos objets d'étude, par le biais d'une combinaison des sources coptes, grecques et latines, transmises par la tradition littéraire, épigraphique et papyrologique. Nous avons associé, d'une part, des billets oraculaires et des papyrus magiques et, de l'autre, des inscriptions isiaques et sérapiques et, encore, des inscriptions grecques trouvées à Rome.
\end{abstract}

Mots clés: Histoire de la Religion ; Papyrologie ; Isis ; Sarapis ; Rome ; Egypte.

Abstract: This study, that had as starting point a recollection of prosopographic data of the Isis cult, has been transformed into a historical-religious research about the religious reality concerned with the belief and veneration of the Egyptian gods, Serapis and Isis, in the Roman context, where their worship reached its apex during the second cent. d.C. Besides ethnic aspects, this study encompasses interpretations on the profile of the followers of these deities, highlighting the participation of freedman, slaves and principally women. One studies here the worship activities, as well as the expectations and conceptions associated with these two deities, through the association of Coptic, Greek and Latin sources, present in literary, papyrologic and epigraphic traditions, using different typologies of documents, as oracular coupons and magical papyri, Serapic and Isiac inscriptions and Greek inscriptions found in Rome.

Keywords: History of Religion; Papyrology; Epigraphy; Isis; Serapis; Rome; Egypt.

Resumo: O presente estudo, que teve como ponto de partida uma coleta de dados prosopográficos do culto a Ísis, transformou-se em uma pesquisa histórico-religiosa sobre a realidade religiosa envolvida na crença e veneração dos deuses egípcios, Serápis e Ísis, no âmbito romano, analisando-se em especial indícios e manifestações destas crenças na cidade de Roma, onde estes cultos alcançaram seu pleno desenvolvimento no séc. II d.C. O estudo engloba interpretações sobre o perfil dos seguidores e seguidoras destas divindades, evidenciando a singular participação de libertos, escravos e sobretudo de mulheres, além da dimensão étnica presente. Práticas cultuais, expectativas e concepções associadas a estas duas divindades são estudadas, por meio de uma combinação entre fontes coptas, gregas e latinas, aportadas pela tradição literária, papirológica e epigráfica. Articulam-se bilhetes oraculares e papiros mágicos gregos; inscrições isíacas e serápicas e inscrições gregas encontradas em Roma.

Palavras-chave: História da religião; Papirologia; Epigrafia; Ísis; Serápis; Roma; Egito.

\footnotetext{
1 “Cultore della Materia” pour l'Histoire des religions, à l'Università di Messina et l'Università di Napoli Due. Le texte suivant a été présenté sous le titre Sexe et statut social des dévots d'Isis et de Sarapis à Rome lors du Colloque international "Les acteurs des cultes isiaques. Identités, fonctions et modes de représentation ", Erfurt, 6-8 mai 2013. Nous remercions Valentino Gasparini pour l'invitation à prendre part à cette remarquable initiative scientifique, Fábio Vergara Cerqueira pour avoir voulu publier ces pages dans une revue scientifique bien connue au niveau international et Xavier Chapel pour la traduction française de nos réflexions historico-religieuses. Nous voulons les dédier à la mémoire de Michel Malaise qui nous a toujours encouragé dans nos recherches : "Manibus, o, date lilia plenis ».
} 
Notre étude, commencée comme un recueil de données prosopographiques, s'est transformée en une recherche historico-religieuse stricto sensu. En effet, la nécessité de comprendre la réalité religieuse qui se trouve au fond de ces données a changé le cap de notre investigation. Pour l'analyse prosopographique, limitée à la ville de Rome, nous avons utilisé les trois recueils essentiels de L. Vidman (1969), M. Malaise (1972a), et L. Bricault $(2005=$ RICIS); pour l'interprétation historico-religieuse, nous avons comparé le patrimoine épigraphique avec les témoignages littéraires et papyrologiques. Nous présentons ici seulement une partie de cette investigation.

Tout le monde sait les nombreux problèmes que pose la prosopographie isiaque et sarapiaque: le manque de données, ce qui va certes à l'avantage des plus problématiques, le fait que la plupart des documents que nous pouvons utiliser sont d'époque impériale, le rôle joué par le hasard des découvertes. Les données que nous pouvons tirer de l'épigraphie sont néanmoins très importantes pour tenter de comprendre la dimension ethnique et sociale de la dévotion à Isis et à Sarapis. De plus, on ne saurait analyser ces données selon une perspective historico-religieuse sans tenir compte des sources littéraires, comme nous allons voir.

Les résultats de notre prosopographie ne s'éloignent pas de ceux que M. Malaise a proposés en 1972: encore une fois, les personnes d'origine étrangère ou les Romains de souche que nous pouvons cataloguer en toute certitude sont numériquement inférieurs à ceux qui échappent à toute tentative de catalogage. Quant à la répartition sociale des fidèles d'Isis et de Sarapis, elle est encore plus incertaine. Les affranchis sont plus nombreux que les esclaves, ce qui ne signifie pas qu'il y avait moins de dévots envers les dieux alexandrins parmi les esclaves. Il faut plutôt songer à la différence des possibilités économiques qui a pu entraîner la différence qualitative des offrandes adressées à Isis et à Sarapis. M. Malaise l'a déjà noté : « En effet, si à l'époque impériale, c'est-à-dire quand la situation est changée et les citoyens ont le droit d'adorer les dieux égyptiens, les esclaves proprement dits n'apparaissent encore que rarement dans les dédicaces, c'est toujours en raison des maigres ressources de la grosse majorité d'entre eux » (Malaise 1972b, p. 89-90).

Si donc une simple dédicace ou une statuette anépigraphe peuvent être le symbole d'une dévotion tout aussi forte que celle de personnes riches provenant des plus hauts niveaux sociaux de la Rome impériale, il sera préférable de focaliser notre attention sur la mentalité plus ou moins transversale qui se trouve à la base de cette dévotion authentique. En outre, le fait que la plupart des inscriptions soient datées entre le II et le $\mathrm{III}^{\mathrm{e}}$ siècle après Jésus-Christ limite toute tentative de reconstruire à travers les données épigraphiques la dynamique chronologique de la diffusion et du succès du culte d'Isis et de Sarapis. Dans un certain sens, les allusions des poètes élégiaques aux cultes d'Isis peuvent être une preuve de la forte présence de cette dévotion dans la Rome d'Auguste, surtout en ce qui concerne la participation des femmes. Les maîtresses de Properce et Ovide sont des isiaques convaincues, elles participent aux célébrations religieuses et sont les représentantes, au niveau féminin, du désir de se mêler à des cérémonies étrangères décrites par des poètes 
qui les ont vues de leurs propres yeux. F. Mora (1990, II, esp. p. 25-26) a mis en évidence la présence numériquement significative des femmes dans les milieux isiaques et sarapiaques à Rome et il faut dire que cette présence est bien attestée au niveau épigraphique. C'est aussi grâce à l'épigraphie que nous savons que les femmes ont joué un grand rôle dans le culte d'Isis et de Sarapis et ce sont elles, par exemple, qui nous font connaître une prophétesse d'Isis.

Tacite offre un témoignage de la persécution de Tibère dirigée à la fois contre les Juifs et les Égyptiens : "Actum et de sacris Aegyptiis ludaicisque pellendis, factumque patrum consultum, ut quattuor milia libertini generis ea superstitione infecta, quis idonea aetas, in insulam Sardiniam veherentur, coercendis illic latrociniis et, si ob gravitatem ceali interissent, vile damnum; ceteri cederent Italia, nisi certum ante diem profanos ritus exuissent " (Tac. Ann. II 85, 4)². Dans ce passage, I'historien distingue entre les individus libertini genus directement exilés en Sardaigne et les ceteri (c'est-à-dire les hommes de naissance libre) qui reçurent la possibilité d'abjurer pour éviter le bannissement. Or, ce témoignage correspond aux résultats de la prosopographie, si nous nous rappelons la présence quantitativement significative de la catégorie des affranchis dans la répartition sociale des fidèles d'Isis et de Sarapis.

Parmi les autres catégories sociales présentes à Rome selon les témoignages épigraphiques, plusieurs clarissimi sont mentionnés plus tard comme dévots des dieux égyptiens; le cas très connu de Vettius Agorius Praetextatus, néocore de Sarapis et dévot de plusieurs divinités, est emblématique. Nous rencontrons aussi des apparitores qui ont adopté le culte d'Isis et de Sarapis : le viator C. Avillius Ligurius Lucanus se déclare prêtre d'Isis. De manière générale, on peut supposer que ceux qui occupaient des postes clés dans les magistratures ou dans l'administration impériale ne sont pas restés étrangers à la dévotion envers Isis et Sarapis, mais les attestations sont peu nombreuses. D'après les documents épigraphiques, ils ont embrassé les cultes des dieux égyptiens à partir de la moitié du $\|^{\mathrm{e}}$ siècle, $c^{\prime}$ est-à-dire à une époque où ces cultes avaient atteint leur plein épanouissement dans la ville de Rome.

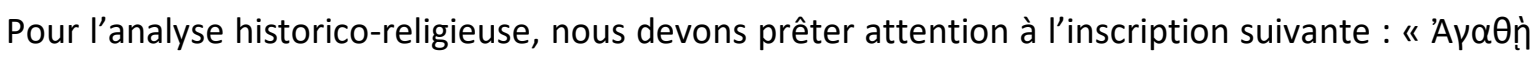

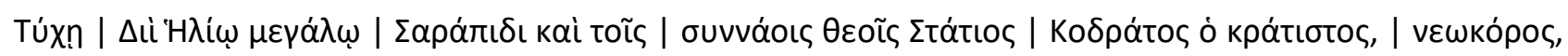

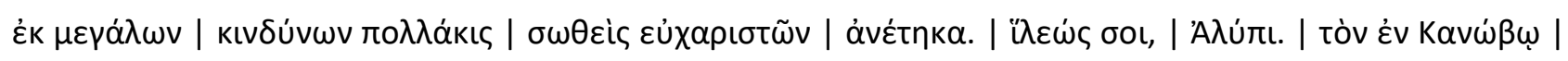

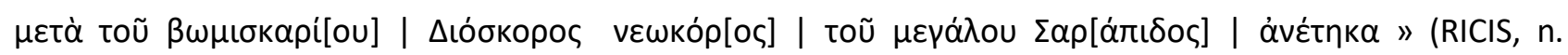

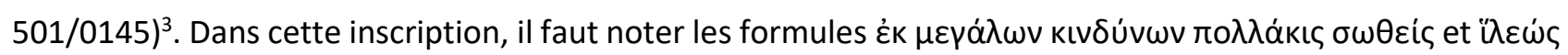

\footnotetext{
${ }^{2}$ " On s'occupa aussi de bannir les cérémonies égyptiennes et judaïques et un sénatus-consulte ordonna que quatre mille affranchis imbus de cette superstition, en âge de porter les armes, fussent transférés en Sardaigne pour y réprimer le brigandage, en considérant que, s'ils succombaient à l'insalubrité du climat, la perte serait légère ; les autres devraient quitter I'Italie si, dans un délai fixé, ils n'avaient pas rejeté leurs rites impies » (trad. P. Wuilleumier).

${ }^{3}$ « À la Bonne Fortune. À l'unique Zeus Hélios grand Sarapis et aux dieux qui partagent le même temple, moi, son excellence Statios Kadratos, néocore, sauvé à plusieurs reprises de grands périls, j'ai consacré (cette colonne) en marque de reconnaissance. Alypios. Qu'ils te soient favorables ! Moi, Dioscoros, néocore du grand Sarapis, j'ai consacré (une statue du type de) celle de Canope, avec son petit autel " (trad. L. Bricault, légèrement remaniée). Pour la formule

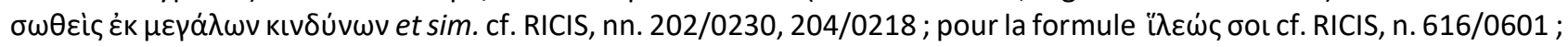
sur Sarapis de Canope cf. RICIS, n. 101/0216.
} 
бol. La seconde formule figure aussi dans un papyrus que l'on tient en général pour magique, mais qui est plutôt un billet oraculaire adressé à Zeus Hélios Grand Sarapis par des fidèles qui demandent au dieu s'il est opportun qu'ils sacrifient, si l'on veut bien tenir compte de la nature de la question et de la clausula, à savoir

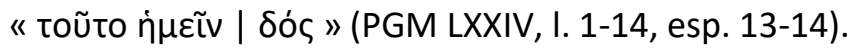

Un papyrus qui, lui, est sûrement du domaine à la magie, indique les modalités à suivre pour obtenir

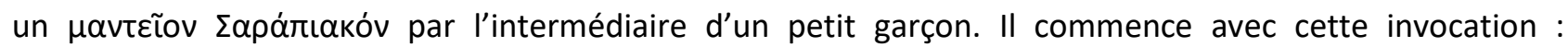

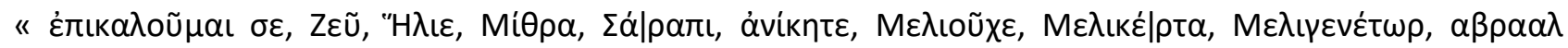

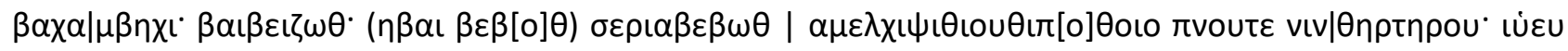

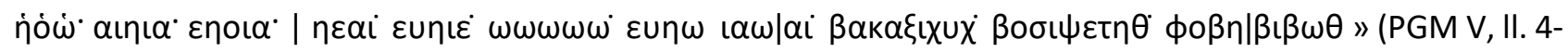

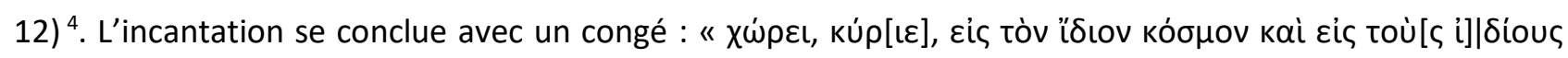

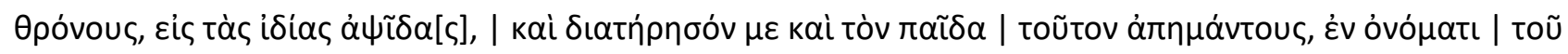
ن்

Tout le monde connaît le pouvoir oraculaire de Sarapis et la technique des billets oraculaires. II suffit de mentionner les papyrus d'Oxyrhynque qui contiennent des questions oraculaires adressées au grand

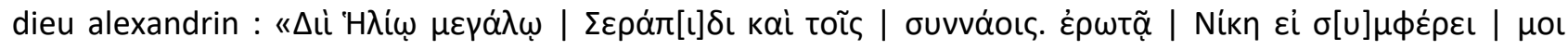

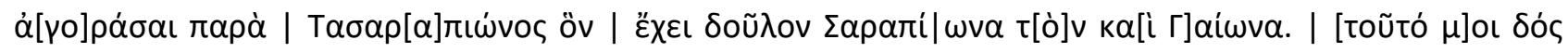

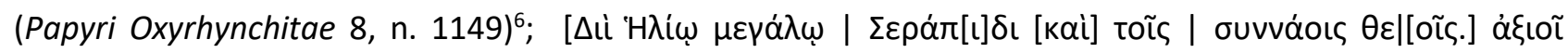

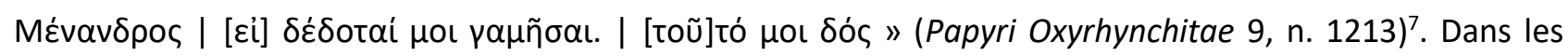
témoignages considérés, il faut noter que Sarapis est presque toujours invoqué comme Zeus Hélios megas. À propos de cette association onomastique, il convient de citer une autre inscription. Dans le pronaos du Mithraeum des Thermes de Caracalla a été trouvé un petit cippe en marbre daté du règne de Caracalla (ou

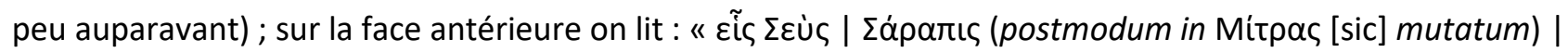

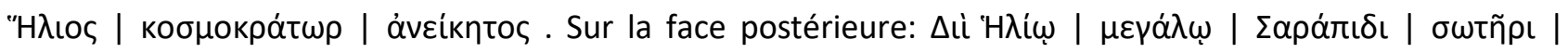

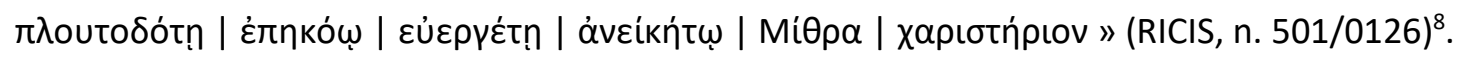

On constatera que le nom de Sarapis a été substitué par celui de Mithra sur la face antérieure. À la lumière de la sensibilité religieuse de l'époque, nous pouvons éliminer la possibilité que Sarapis ait été l'objet d'une damnatio memoriae et il vaut mieux penser à une substitution de l'un par l'autre à cause de leur qualité

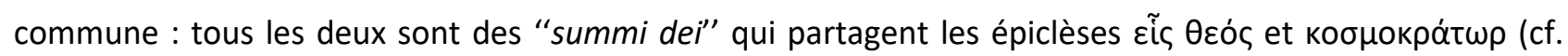

\footnotetext{
${ }^{4}$ "Je t'invoque, Zeus, Hélios, Mithra, Sarapis, invaincu, Meliouchos, Melikertes, Meligenetor voces magicae ».

${ }^{5}$ « Va, Seigneur, vers ton cosmos, vers tes trônes, tes voûtes célestes, et garde-moi et cet enfant en toute sécurité, au nom de Dieu très haut voces magicae "; cf. Sanzi 2010)

${ }^{6}$ « À Zeus Hélios grand Sarapis et aux dieux qui partagent le même temple, Nikè demande si je dois aller au marché chez Tasarapion, celui qui a un esclave Sarapion, appelé aussi Gaionas, donne-moi ce billet oraculaire. »

${ }^{7}$ « À Zeus Hélios grand Sarapis et aux dieux qui partagent le même temple, Ménandre demande si je dois me marier, donne moi ce billet oraculaire »

${ }^{8}$ « Zeus [[Sarapis]] Hélios, maître du monde, invincible, ne font qu'un. À Zeus Hélios grand Sarapis, sauveur, qui procure la richesse, qui écoute les prières, bienfaisant, invincible, Mithra, en marque de reconnaissance » (trad. L. Bricault).
} 
Peterson 1926, p. 227-240, esp. 239-240). Au sujet de cette inscription, Ugo Bianchi a noté que l'appellation de Sarapis comme dieu de l'Olympe en souligne encore plus la qualité de deus summus, universel et capable de soutenir l'univers entier, c'est-à-dire le grand cosmos qui est soumis au pouvoir presque absolu de Sarapis. En outre, le dieu alexandrin est nommé Hélios : une figure divine sur laquelle la sensibilité religieuse hellénistique et romaine avait fait concentrer l'essence de la divinité. Le savant italien conclut en disant:

"Nella nostra iscrizione ... Serapide è piuttosto il sostantivo, mentre Zeus ed Helios hanno funzioni di apposizioni, che qualificano la grandezza del dio. Il quale è appunto

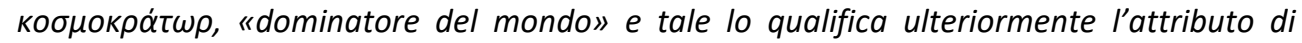

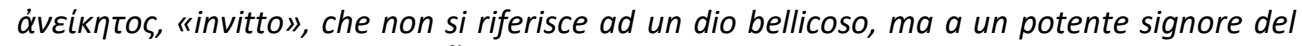
mondo. Infine il dio è detto eís, "uno solo", per affermare la sua eccellenza, unica: una proclamazione di fede enoteistica » (Bianchi 1975, p. 247).

Il faut revenir maintenant aux billets oraculaires pour souligner la continuité de la sensibilité religieuse entre l'Égypte hellénistique et l'Égypte copte. Les archéologues et les épigraphistes qui ont travaillé dans la ville d'Antinoé ont démontré la fonction oraculaire du sanctuaire consacré au saint martyr Colluthe. De ce sanctuaire, en effet, provient un grand nombre de billets oraculaires dont la structure est identique à

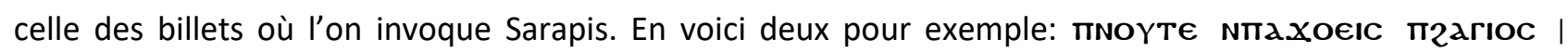

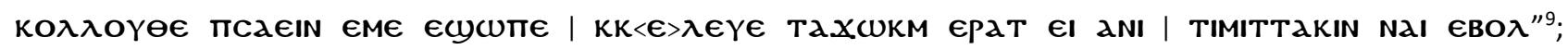

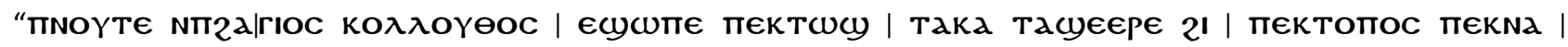
NaTa2OC | €KaOYOcy|q€ $T^{10}$. On relèvera que le mot copte pnoute, "Dieu », a été utilisé comme vox magica dans les papyrus cités relatifs à l'oracle de Sarapis.

Quant à la conclusion théologique des textes d'Oryrhynque, elle se fonde sur la qualité du pouvoir cosmique de Sarapis qui garantit à la fois la véridicité de la réponse et la confiance en sa vertu apotropaïque. Naturellement, dans cette dimension mantique, la figure du prophète revêt une grande importance.

À ce propos, il sera utile de mentionner une inscription trouvée dans le sous-sol de Santa Maria in Via Lata, qui se rattache à I'Iseum Campense et est datée du 6 mai 146 apr. J.-C. :

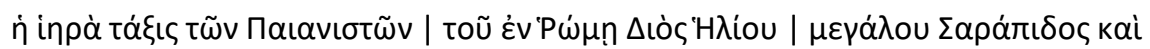

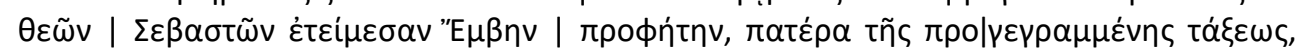

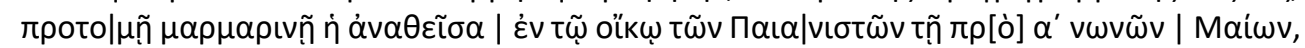

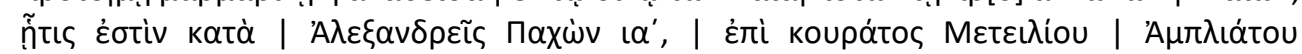

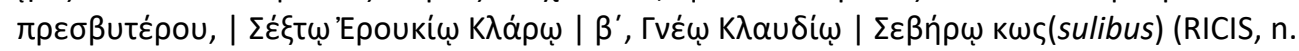
$501 / 0118)^{11}$.

\footnotetext{
${ }^{9}$ " Dieu de mon Seigneur, saint Colluthe le vrai médecin, si tu me commandes de laver mes pieds, donne-moi ce billet oraculaire »; cf. Donadoni 1964).

10 "Dieu de saint Colluthe, si tu veux que je mette ma fille dans ton topos, ton désir sera en fonction de votre volonté »; cf. Papini 1985, esp. 249-250).

11 «La confrérie sacrée des Péanistes de Zeus Soleil grand Sarapis à Rome et des dieux Augustes a honoré Embès, prophète, père de la confrérie susnommée, en dédiant son buste en marbre dans l'oikos des Péanistes, la veille des nones de Mai, qui est le 11 du mois Pachôn selon le calendrier d' Alexandrie, sous le curatorat de Méteilios Ampliatos I'ancien, Sextos Éroukios Klaros pour la seconde fois et Gnaios Klaudios Sèvéros étant consuls ». Les Péanistes,
} 
Il faut souligner, d'un côté, l'honneur rendu au prophète et père du collège des Péanistes dédié à Sarapis qui est qualifié de megas, et de l'autre, le nom du dieu flanqué par ceux de Zeus et Hélios, comme dans les deux textes d'Oxyrhynque. Dans cette inscription est évidente la volonté de souligner la prérogative oraculaire de Sarapis, si c'est un prophète du dieu qui est honoré. Pourtant, il ne sera pas inutile de rapporter la réponse prononcée directement par le dieu alexandrin au roi Nicocréont qui voulait connaître quel type

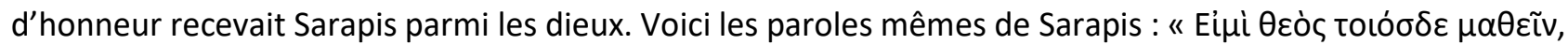

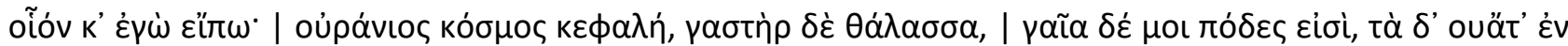

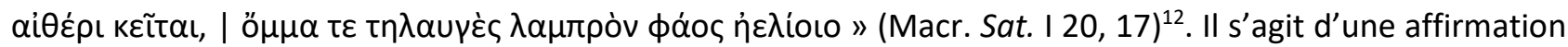
catégorique prononcée directement par le dieu.

Il paraît intéressant de prêter attention à l'Éloge des saints Pierre et Paul, un texte rédigé directement en copte et daté des VII ${ }^{\mathrm{V}} \mathrm{VIII}{ }^{\mathrm{e}}$ s. apr. J.-C. Cet éloge contient une Vie de saint Marc qui mentionne Sarapis. Nous avons déjà présenté le texte dans le deuxième numéro de Bibliotheca Isiaca et il nous suffira de citer ici le moment le plus significatif qui suit le martyre de saint Marc : la statue de Sarapis est posée sous le Tétrapyle, mais le démon qui l'habite déclare qu'il ne peut plus demeurer dans la statue parce que le sang de l'apôtre a purifié la ville entière. Le démon sort immédiatement du simulacre et l'idole perd l'usage de la parole. Dorénavant, malgré toutes les sollicitations des païens, Sarapis ne parlera plus. Nous avons déjà supposé que cet éloge contient un souvenir précis de l'ancien culte de Sarapis, en particulier de sa dimension oraculaire liée à la summa potestas du dieu :

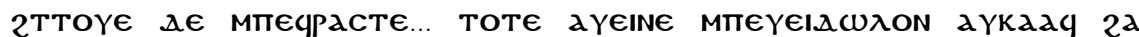

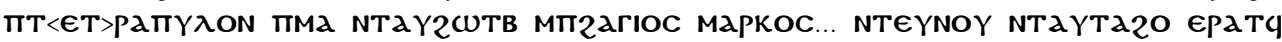

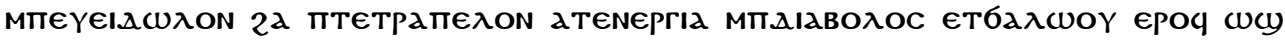

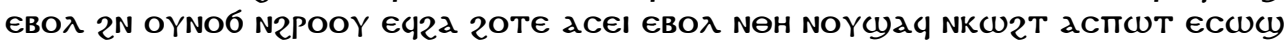

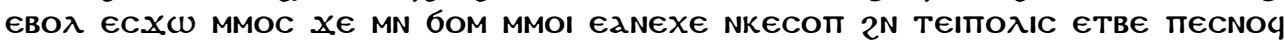

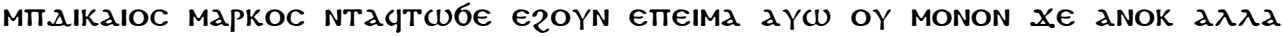

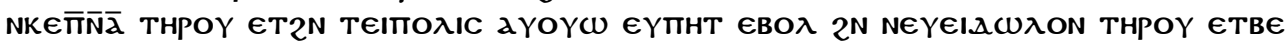
пECNOG MாEIPCUME N.AIKaIOC ETMMAY ETEIAH TENTAqTaMIE TENEICUT THPN

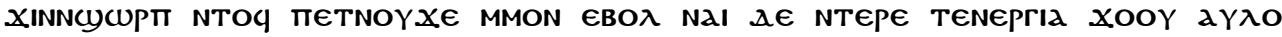

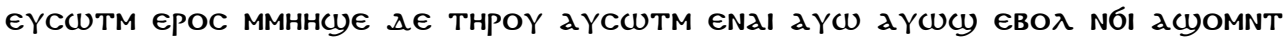

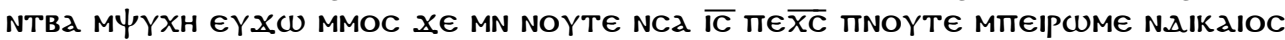

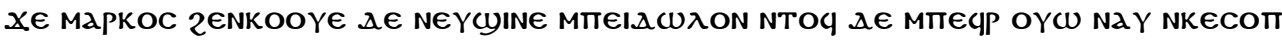
(Depuydt 1993, I, p. 129) ${ }^{13}$.

hymnodes peut-être d'origine alexandrine, formaient une confrérie professionnelle qui avait son propre oikos dans l'enceinte de l'Iseum Campense.

12 « Je suis un dieu qui doit être connu tel que moi, je veux le dire. Ma tête est le cosmos céleste, le ventre la mer, la terre mes pieds, les oreilles sont posées dans l'éther, les yeux brillent de loin la lumière brillante du soleil ».

${ }^{13}$ Depuydt 1993, 129: « Le lendemain à l'aube... ils prirent leur idole et la mirent sous le tetrapylon, le lieu où ils avaient tué Saint Marc... quand ils érigèrent leur idole sous le tetrapylon, la puissance du diable, qui habitait là bas, cria d'une voix forte et effrayante, sortit comme une flamme de feu et s'enfuit en criant et en disant: "Je ne peux pas rester dans cette ville parce que le sang de Marc le juste est ici, et pas seulement moi, mais tous les autres esprits qui étaient dans cette ville ont fui de leurs idoles à cause du sang de ce juste (en disant) : " Celui en effet qui a créé le père de nous tous, dès le début, c'est lui qui nous fait partir ". Lorsque la force du démon eut dit cela, ils cessèrent de l'entendre. Toute la foule avait entendu ces choses et trente mille âmes s'exclamèrent en disant: "Il n'y a pas de Dieu en dehors de JésusChrist, le Dieu de cet homme juste, Marc. » D'autres, cependant, interrogeaient l'idole, mais elle ne leur répondit plus ». 
Au milieu du $\|^{e}$ siècle apr. J.-C., Aelius Aristide avait bien souligné la dimension hénothéiste et sotériologique de Sarapis. Ajoutons que dans l'Oratio in Sarapidem, le salut dont le dieu se porte garant se fonde sur sa summa potestas :

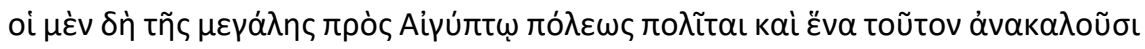

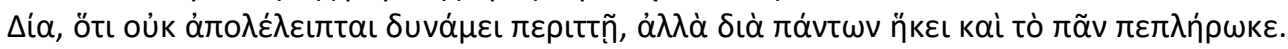

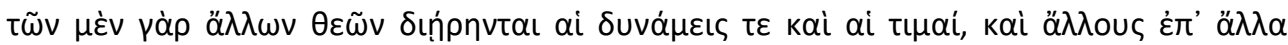

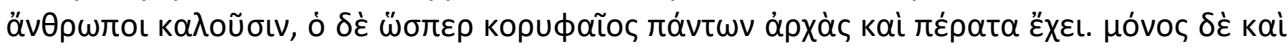

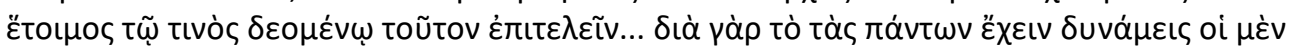

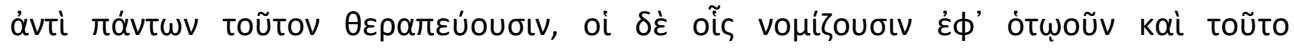

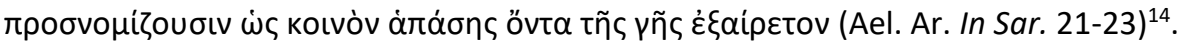

C'est bien un tel Sarapis qui a dû être présent à l'esprit de Statios Kadratos, le néocore sauvé par le dieu auquel il consacre une colonne, comme nous l'avons vu plus haut. Nous pouvons retrouver la même

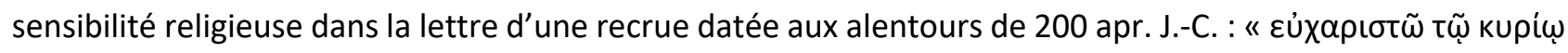

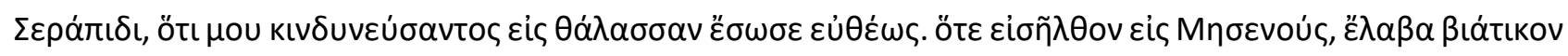

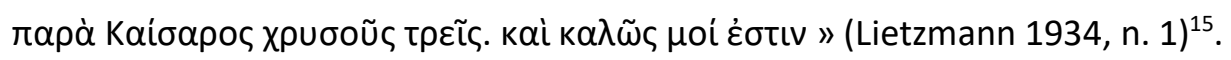

Il faut noter que tant dans cette lettre que dans l'inscription de Kadratos, le verbe utilisé est $\sigma \hat{\varphi} \zeta \omega$. Ce verbe apparaît à l'aoriste actif s'il concerne le dieu et au passif s'il concerne celui qui fait l'objet de la bienveillance de Sarapis : à l'intervention bénéfique du dieu correspond donc la totale dépendance des fidèles qui sont sauvés exclusivement en vertu de cette intervention bienveillante.

Nous pouvons percevoir l'écho d'une même sensibilité religieuse dans une inscription qui provient du Dolocenum de l'Aventin : I(ovi) o(ptimo) s(ancto) p(raestantissimo) D(olicheno) | et lunoni sanctae, | Herae, Castori(bus) | et Apollini conservato|ribus Thyrsus | pro salute patroni sui | et sua suorumque | iussu numinis eorum | aram d(edit) | salvis candidatis | huius loci... (CCID, n. 383) ${ }^{16}$. En ce qui concerne la qualité de la potestas du dieu et des attentes sotériologiques de nature intra-cosmique qu'il assure, cette inscription est paradigmatique. II s'agit de la consécration d'un autel dédié à Jupiter Dolichenus, sainte Junon, Héra, les Castors et Apollon pour obéir à un ordre du numen de ces divinités et à la suite d'un péril dont les candidats du sanctuaire ont été sauvés. Ces divinités sont tutélaires d'une sotériologie au niveau intra-cosmique. Nous sommes ici dans un milieu dolichénien et pourtant les divinités qui flanquent ce Jupiter soulignent encore

\footnotetext{
${ }^{14}$ « Les citoyens de la grande cité au bord de l’Égypte (sc. Alexandrie) vont jusqu'à l'invoquer du nom de Zeus unique, parce qu'il ne lui est pas inférieur par sa puissance extraordinaire, mais qu'il passe partout et a rempli l'univers. En effet, les pouvoirs et les honneurs des autres dieux sont répartis, et les hommes invoquent chaque foi un dieu différent dans un but différent. Mais lui, comme un coryphée, il détient les principes et les fins de toutes choses, et lui seul est prêt à accomplir ce dont on a besoin... Parce qu'il détient les pouvoirs de tous les dieux, les uns le vénèrent à la place de tous les dieux, les autres, outre les dieux auxquels ils croient en n'importe quelle circonstance, croient aussi en celui-ci, dans l'idée qu'il est un dieu spécial commun à toute la terre » (trad. J. Goeken).

15 « Je remercie vraiment le Seigneur Sarapis, parce que lorsque j'étais en danger en pleine mer, il m'a immédiatement sauvé. Quand je suis arrivé à Misène, j'ai pris de César un salaire de trois pièces d'or. J'ai été vraiment chanceux! ».

${ }^{16}$ «En l'honneur de Jupiter très bon saint excellent Dolichénus et sainte Junon, Héra, Apollon et les Castors et Apollon conservateurs, Thyrse, pour la bonne santé de son patron, la sienne et celle de sa famille, par ordre de leurs numen, a offert l'autel, les candidats de ce lieu étant saufs... ".
} 
une fois la qualité sotériologique du dieu. Dans cette inscription, l'ablatif salvis candidatis huius loci laisse supposer que le groupe des divinités qui sauvent et qui sont invoquées en tant que conservatores, - titre qui, à plusieurs reprises, qualifie Jupiter Dolichénus -, accentue encore la dimension sotériologique assurée par le dieu kosmokrator et omnipotens.

La bienveillance de Sarapis se manifeste au niveau tant individuel qu'universel. A ce propos, il

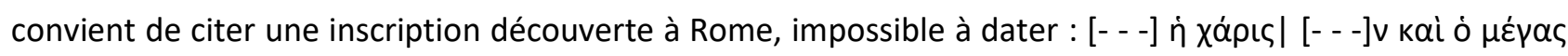

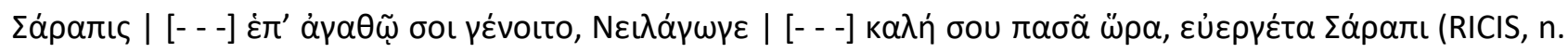
$501 / 0215)^{17}$.

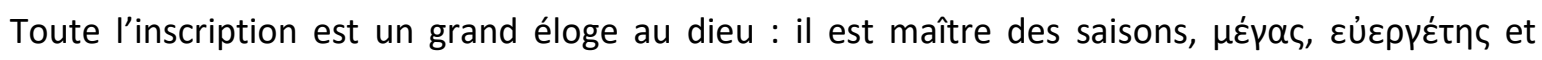
$\mathrm{N} \varepsilon \lambda_{\lambda} \alpha{ } \omega$ wpos. De toute évidence, un Sarapis si puissant est capable de garantir la protection de tout être vivant. Aelius Aristide semble faire parler cette inscription, quand il loue le dieu en disant :

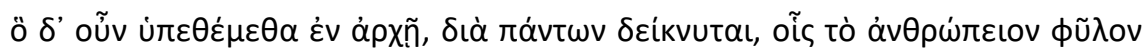

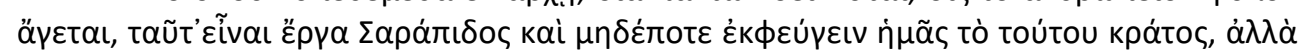

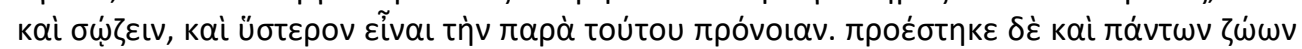

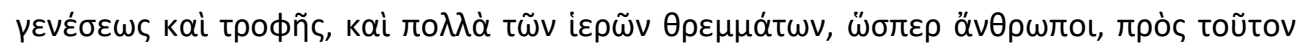

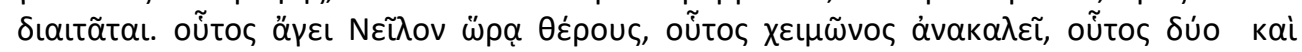

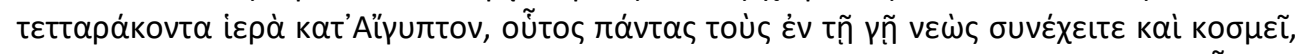

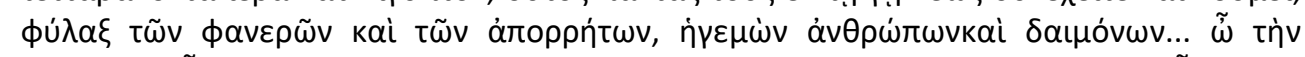

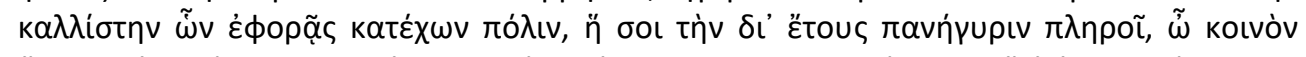

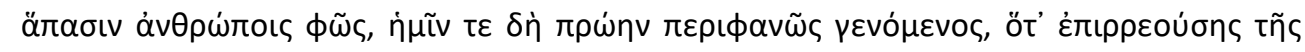

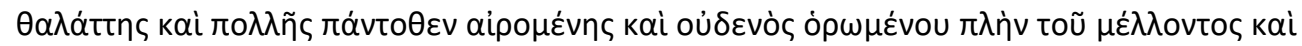

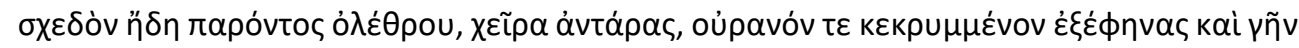

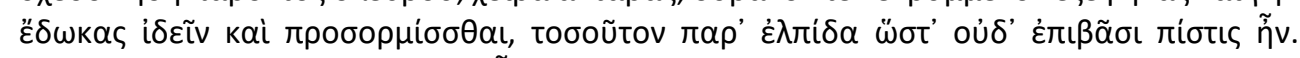

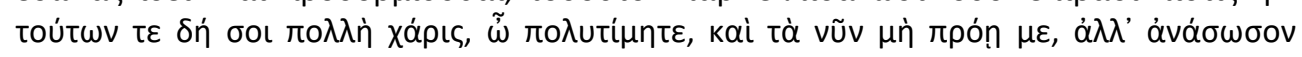

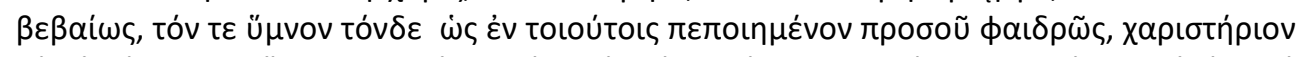

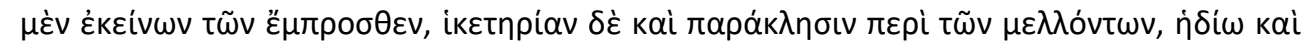

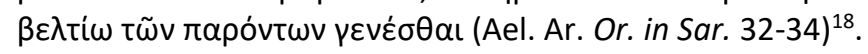

\footnotetext{
17 «[...] la reconnaissance [...] et le grand Sarapis [...] que ceci soit pour toi dans une bonne intention, toi qui conduis le Nil. [...] Chacune de tes saisons ( ?) est belle, bienfaisant Sarapis » (trad. L. Bricault). Pour le titre de Neilagogos cf. RICIS 304/0611.

18 «Quoi qu'il en soit, ce que nous suggérions pour commencer, tout le prouve : les œuvres de Sarapis sont ce qui guide la race humaine, nous n'échappons jamais au pouvoir de celui-ci, et sa providence consiste à assurer aussi le salut plus tard. Il préside en outre à la naissance et à la subsistance de tous les êtres, et nombreuses sont les créatures sacrées qui, comme des hommes, séjournent auprès de lui. C'est lui qui amène le Nil à la saison estivale, c'est lui qui le rappelle en hiver, c'est lui qui dirige et orne quarante-deux sanctuaires en Égypte, ainsi que tous les temples de la terre, gardien de ce qui est visible et de ce qui est secret, guide des hommes et des divinités... Ô maître de la plus belle des cités à être sous ta garde, celle qui célèbre pour toi la panégyrie annuelle ! Ô lumière commune à tous les hommes, toi qui nous es récemment apparu! Au moment où la mer grosse nous submergeait et se soulevait de tous côtés, et où l'on ne voyait rien si ce n'est la mort qui devait arriver et qui était déjà presque là, tu levas la main, tu fis apparaître le ciel qui était caché et tu nous accordas de voir la terre et d'aborder, de façon tellement inespérée que nous n'y croyions même pas après avoir débarqué. C'est pourquoi j'ai beaucoup de reconnaissance à ton égard, ô toi que l'on honore beaucoup, et maintenant, ne m'abandonne pas, mais délivre-moi pour de bon et accueille avec joie cet hymne que j'ai composé dans de telles circonstance, afin de manifester ma reconnaissance pour ces faits passés, et afin de te supplier et de t'implorer au sujet de l'avenir, pour qu'il soit plus heureux et meilleur que le présent » (trad. J. Goeken).
} 
Venons-en maintenant à une inscription qui se révèle très intéressante si on la compare avec les sources littéraires. II s'agit d'un autel en marbre, trouvé à Rome dans la regio IX « Iseum et Serapeum », daté entre 71 et 79 apr. J.C. et qui doit justement provenir de l'Iseum Campense. En voici le texte : Isidi sacr(um) | Crescens | Caesaris | Vespasiani | ex visu posuit (RICIS, n. 501/0116) ${ }^{19}$.

Deux choses sont à souligner : la première, c'est le fait que le dédicant est un esclave de Vespasien (selon une autre interprétation, il serait un esclave de Titus) ; la seconde, c'est l'utilisation de la formule ex visu. Tacite et Suétone ont conservé l'épisode des miracles opérés par Vespasien pendant son séjour à Alexandrie. Tacite raconte :

E plebe Alexandrina quidam oculorum tabe notus genua eius advolitur, remedium caecitatis exposcens gemitu, monitu Serapidis dei, quem dedita superstitionibus gens ante alios colit, precabaturque principem, ut genas et oculorum orbes dignaretur respergere oris excremento. Alius manum aeger eodem deo auctore, ut pede ac vestigio Caesaris calcaretur, orabat (Tac. Hist. IV 81, 1) $)^{20}$.

Et voici la version de Suétone :

"E plebe quidam luminibus orbatus, item alius debili crure sedentem pro tribunali pariter adierunt orantes opem valitudini demonstratam a Serapide per quietem: restituturum oculos, si inspuisset; confirmaturum crus, si dignaretur calce contingere" (Suet. Vesp. 7) $)^{21}$.

L'empereur est incrédule et ne voudrait pas condescendre aux désirs des deux malades, mais sous la pression des adulateurs, il se rend aux prières et la prophétie de Sarapis se réalise. Durant le même séjour, Vespasien consulte Sarapis dieu oraculaire. Il faut citer encore une fois les deux historiens romains. Tacite :

Altior inde Vespasiano cupido adeundi sacram sedem, ut super rebus imperii consuleret: arceri tempio cunctos iubet. Atque ingressus intentusque numini respexit pone tergum e primoribus Aegyptiorum nomine Basiliden, quem procul Alexandria plurium dierum itinere et aegro corpore detineri haud ignorabat. Percunctatur sacerdotes, num illo die Basilides templum inisset, percunctatur obvios, num in urbe visus sit; denique missis equitibus explorat, illo temporis momento octoginta milibus passuum afuisse: tunc divinam speciem et vim responsi ex nomine Basilidis interpretatus est (Tac. Hist. IV 82, 1-2)22.

\footnotetext{
${ }^{19}$ « Consacré à Isis. Crescens (esclave) de César Vespasien a fait cette offrande à la suite d'une vision » (trad. L. Bricault). 20 « Un habitant d'Alexandrie, un homme du peuple, bien connu pour la sanie qui lui rongeait les yeux, se jette à ses genoux, implorant en gémissant un remède à sa cécité, sur le conseil de Sarapis, dieu que ce peuple adonné aux superstitions vénère plus que tous les autres; il suppliait le prince de daigner lui humecter avec sa salive les paupières et les orbites ; un autre dont la main était estropiée, suppliait César, à l'instigation du même dieu, de fouler cette main avec la plante de son pied » (trad. H. Le Bonniec).

${ }^{21}$ « Deux hommes du peuple, dont l'un était aveugle et l'autre avait une jambe infirme, vinrent le trouver en même temps, pendant qu'il siégeait sur son tribunal, et le supplièrent de faire, pour les guérir, ce que Sarapis leur avait indiqué en songe : il rendrait à l'aveugle l'usage de ses yeux, s'il les humectait de salive ; à la jambe infirme, sa vigueur, s'il daignait la toucher avec le pied » (trad. H. Ailloud).

${ }^{22}$ «Vespasien n'en souhaita que plus profondément de pénétrer dans la demeure sacrée du dieu, pour le consulter sur les affaires de l'Empire ; il interdit à tout le monde l'accès du temple. Il y entra et, l'esprit occupé par la pensée du dieu, il aperçut derrière son dos un des notables égyptiens, nommé Basilidès, dont il n'ignorait pas qu'il se trouvait loin d'Alexandrie, à plusieurs jours de marche et immobilisé par la maladie. Il demande aux prêtres si Basilidès est venu au
} 
Suétone :

Hic cum de firmitate imperii capturus auspicium aedem Serapidis summotis omnibus solus intrasset ac propitiato multum deo tandem se convertisset, verbenas coronasque et panificia, ut illic assolet, Basilides libertus obtulisse ei visus est; quem neque admissum a quoquam et iam pridem propter nervorum valitudinem vix ingredi longeque abesse constabat. Ac statim advenere litterae fusas apud Cremonam Vitelli copias, ipsum in Vrbe interemptum nuntiantes" (Suet. Vesp. 7) ${ }^{23}$.

On peut constater que la prédiction du dieu se manifeste à travers l'escamotage le subterfuge de la vision. Si Tacite justifie les événements de manière rationnelle, Suétone, qui peut en partie dépendre de lui, semble donner voix à la composante "orientale" de la politique de Vespasien. Si on se rappelle que l'empereur et son fils Titus, avant de célébrer leur triomphe, passèrent la nuit dans l'Iseum Campense (ce dont témoigne clairement Flavius Josèphe $=$ Bell. Iud. VII $123^{24}$ ) et que la ville d'Alexandrie et ses dieux ont joué un rôle très important dans la légitimation de la dynastie flavienne (comme il ressort de la formule d'acclamation avec laquelle le peuple de la ville a accueilli le général, conservée dans le Papyrus Fouad, n. 8, II. $14-15^{25}$ ), il semble naturel d'imaginer le lien que Vespasien et sa propagande ont établi avec Isis et Sarapis.

Nous pouvons considérer aussi l'épisode de la rencontre entre Apollonius de Tyane et Vespasien dans le Serapeum d’Alexandrie narré par Philostrate. Arrivé au sanctuaire, le général

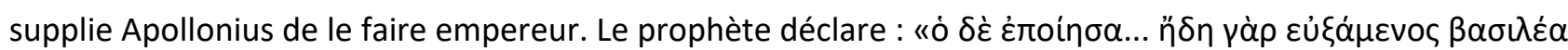

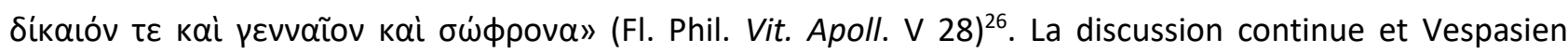
demande des conseils. Apollonius alors s'adresse à Jupiter Capitolin et

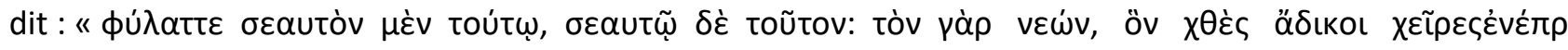

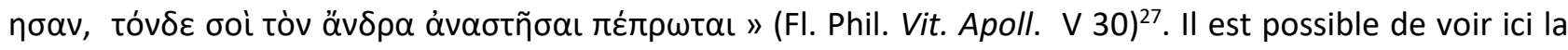
relation qui s'instaure entre Vespasien, la sauvegarde du mos maiorum et, en même temps, le caractère

temple ce jour-là ; il demande aux passants si on l'a vu dans la ville; enfin il envoie des cavaliers et s'assure qu'au moment où il l'avait vu, il était à quatre-vingts milles de là ; alors il expliqua l'apparition comme envoyée par le dieu et donna au nom de Basilidès le sens d'une prophétie " (trad. H. Le Bonniec).

${ }^{23}$ « Là (sc. en Alexandrie d’Égypte), voulant demander à Sarapis si son pouvoir était bien assuré, il entra dans le temple de ce dieu, tout seul, en renvoyant toute sa suite, et, quand il se retourna enfin, après avoir longuement prié le dieu, il crut voir l'affranchi Basilidès lui offrant, suivant l'usage du pays, de la verveine, des couronnes et des gâteaux ; or, il était avéré que personne n'avait introduit cet homme, que, d'ailleurs, depuis longtemps, par suite de rhumatismes, il pouvait à peine marcher et se trouvait fort loin de là. Puis, aussitôt après, arriva une lettre annonçant que les troupes de Vitellius avaient été battues à Crémone, et lui même tué à Rome » (trad. H. Ailloud).

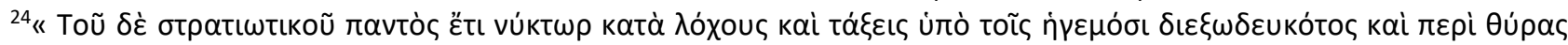

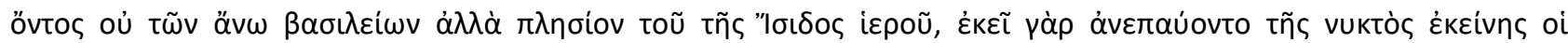

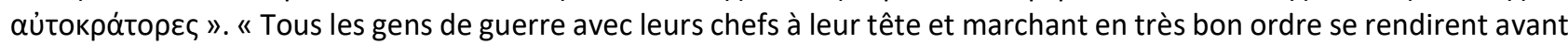
le jour auprès des portes, non pas du palais d'en haut, mais du temple d'Isis où les deux princes avaient passé la nuit »(trad. J.-A.-C. Buchon).

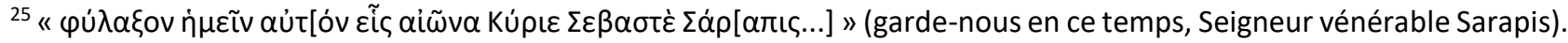
${ }^{26}$ " Je vous ai déjà (fait empereur) le jour où j'ai demandé aux dieux un prince juste, noble et vertueux » (cf. Derchain Hubaux 1953).

${ }^{27}$ « Garde-toi toi-même pour l'homme que voici et garde-le pour toi. Car c'est lui-même qui est destiné à rebâtir en ton honneur le temple qui hier même a été réduit en cendres par des mains malfaisantes ». 
exceptionnel d'un empereur choisi directement par les dieux de Rome. Par ailleurs, si l'empereur se laisse adorer comme un dieu en Orient, à Rome il suit la politique prudente d'Auguste. Mais il y a une grande différence entre Vespasien et Auguste - et c'est la généalogie : la famille du premier n'est pas enracinée dans la Troade, n'a pas de liens directs avec les dieux. II est donc possible que Vespasien ait aussi cherché une légitimation à travers les dieux alexandrins, légitimation qui par ailleurs ne constituait aucune prévarication du sénat. À vrai dire, dans le témoignage de Flavius Josèphe, c'est la description du triomphe qui joue le grand rôle. L'apparition de la façade de l'Iseum Campense dans la numismatique officielle peut être interprétée d'une part comme la démonstration de la faveur accordée à Vespasien par Isis et Sarapis et, de l'autre, comme la commémoration de la nuit passée dans ce sanctuaire avant de célébrer le triomphe. C'est dans ce nouveau climat que nous pouvons situer l'inscription de Crescens, un climat instauré par Vespasien qui a pu nourrir une piété personnelle envers les dieux d'Alexandrie à cause de de l'appui reçu.

Dans notre inscription, c'est un esclave de l'empereur qui a obtenu une vision d'Isis, vision qui sans doute est le fruit de la bienveillance d'une déesse qui n'hésite jamais à se montrer quand il faut aider ses fidèles. La provenance de l'inscription est significative : encore une fois, c'est l'Iseum Campense, c'est-à-dire le sanctuaire isiaque lié à Vespasien et Titus. Pour reconstruire la sensibilité religieuse qui a poussé Crescens à offrir cette inscription, nous pouvons citer un passage des Métamorphoses d'Ovide. Il s'agit de l'apparition de la déesse Isis à une femme enceinte nommée Telethusa. La jeune femme est remplie de terreur, parce qu'elle sait bien que son mari n'acceptera jamais d'élever une fille. Elle prie alors Isis et reçoit une grande et douce vision: la déesse en majesté apparaît avec son cortège et fait une promesse: "Pars o Telethusa mearum, / Pone graues curas mandataque falle mariti; / nec dubita, cum te partu Lucina leuarit, / tollere quidquid erit. Dea sum auxilias opemque / exorata fero; nec te coluisse quereris / ingratum numen" (Ov. Met. IX 696-700) $)^{28}$.

À ce passage d'Ovide fait écho une inscription gravée sur une pierre retrouvée à Rome : ISIPDM | Isidi salutari | pro sal(ute) Q. Vergili | Modesti Cassia mater | v(oto) s(oluto) d(edicavit) (RICIS, n. 501/0151)29. Il s'agit évidemment de l'accomplissement du vœux d'une mère à la déesse Isis qui est qualifiée de salutaris, épiclèse attestée en latin pour la déesse uniquement dans cette inscription. Une telle sensibilité dévotionnelle n'est évidemment pas l'exclusivité de la grande ville de Rome et pour s'en convaincre, il suffit de rappeler les titres $\sigma \omega \zeta$ گoũ $\sigma \alpha$ et $\sigma \omega ́ \tau \varepsilon เ \rho \alpha$ avec lesquels la déesse est invoquée à plusieurs reprises. II ne faut pas non plus oublier un passage des Métamorphoses d'Apulée. Le protagoniste du roman, Lucius, initié aux

\footnotetext{
${ }^{28}$ « Teléthuse, toi que je compte au nombre de mes adoratrices, dépose le fardeau de tes peines et dérobe-toi de ton époux ; n’hésite pas ; quand Lucine t’aura délivrée, élève ton enfant, quel qu'en soit le sexe. Je suis une déesse secourable et je viens en aide à qui m'implore ; tu n'auras pas à te plaindre d'avoir honoré une divinité ingrate " (trad. G. Lafaye).

${ }^{29}$ « (.[ ?].) À Isis qui guérit, pour la santé de Quintus Vergilius Modestus, sa mère Cassia a dédié ceci en acquittement de son vœu » (trad. L. Bricault).
} 
mystères d'Isis, apparaît à la foule des fidèles et prie la grande déesse avec des mots très proches de ceux des arétalogies:

Tu quidem sancta et humani generis sospitatrix perpetua, semper fovendis mortalibus munifica, dulcem matris adfectationem miserorum casibus tribuis. Nec dies nec quies nulla ac ne momentum quidem tenue tuis transcurrit beneficiis otiosum, quin mari terraque protegas homines et depulsis vitae procellis salutarem porrigas dexteram, qua fatorum etiam inextricabiliter contorta retractas licia et Fortunae tempestates mitigas et stellarum noxios meatus cohibes. Te superi colunt, observant inferi, tu rotas orbem, luminas solem, regis mundum, calcas tartarum. Tibi respondent sidera, redeunt tempora, gaudent numina, serviunt elementa. Tuo nutu spirant flamina, nutriunt nubila, germinant semina, crescunt germina. Tuam maiestatem perhorrescunt aves caelo meantes, ferae montibus errantes, serpentes solo latentes, belvae ponto natantes (Ap. Met. XI 25). ${ }^{30}$

Au niveau de l'histoire des religions, les vers d'Ovide et les mots d'Apulée nous aident ainsi à intégrer le témoignage épigraphique laissé par Cassia dans la sensibilité religieuse qui l'a produite.

Mais il n'y a pas qu'Isis à être une déesse qui sauve et qui guérit : Sarapis aussi, nous l'avons vu, est très généreux envers ses fidèles. À ce propos et pour conclure, nous allons citer une dernière inscription, trouvée à Rome et datée entre 192 et 212.

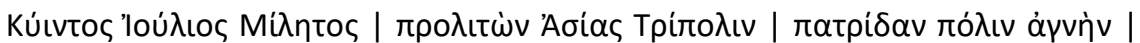

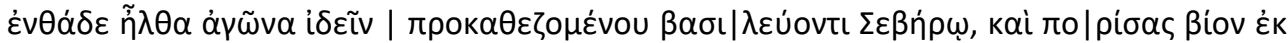

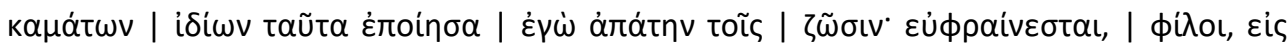

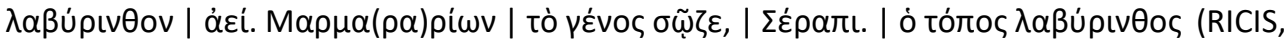
n. 501/0207) $)^{31}$.

II s'agit d'un marbrier qui a fait fortune à Rome. Il convient de souligner deux choses dans cette inscription. La première concerne l'invocation à Sarapis : encore une fois, nous pouvons constater l'utilisation du verbe $\sigma \omega \grave{\zeta} \zeta \omega$ dans un contexte relatif à une dimension sans doute intramondaine. La deuxième concerne le sens du mot $\lambda \alpha \beta u ́ p ı v \theta o s$.

Avant d'analyser le sens de ce mot, il faut concentrer notre attention sur le verbe $\varepsilon u ́ \varphi \rho \alpha i ́ v \varepsilon \sigma \theta \alpha$, à lire $\varepsilon u ̉ \varphi \rho \alpha i ́ v \varepsilon \sigma \theta \varepsilon$. Sur un bloc de marbre retrouvé à Paros daté entre le III et le IVe siècle apr. J.-C., à

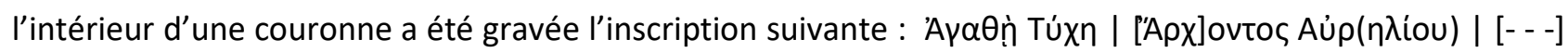

\footnotetext{
30 « Sainte ! Toi qui veilles sans te lasser sur le salut du genre humain, toujours prodigue envers les mortels des soins qui les raniment, tu dispenses à l'infortune la douce tendresse d'une mère. II n'est ni jour, ni nuit, ni instant fugitif que tu laisses passer sans le marquer de tes bienfaits, sans protéger les hommes sur mer et sur terre, sans chasser loin d'eux les orages de la vie, sans leur tendre la main secourable qui dénoue les réseaux les plus inextricables de la fatalité, calme les tempêtes de la fortune et maîtrise le cours funeste des étoiles. Les dieux du ciel te rendent hommage, les dieux de l'enfer te respectent ; tu meus le monde sur son axe, tu allumes les feux du soleil, tu gouvernes l'univers, tu foules de tes pieds le Tartare. Les astres sont dociles à ta voix, les saisons reviennent à ta volonté, les dieux se réjouissent à ta vue, les éléments sont à tes ordres. Tu fais un geste et les brises s'animent, les nuages s'enflent, les semences germent, les germes grandissent. Ta majesté remplit d'un saint effroi les oiseaux qui parcourent le ciel, les animaux qui errent par les montagnes, les serpents qui se cachent sous terre, les monstres qui nagent dans l'Océan » (trad. P. Vallette).

${ }^{31}$ « Kointos loulios Milètos, ayant quitté Tripolis en Asie, cité sainte (qui est) ma patrie. Je suis venu ici pour assister à un concours présidé par l'empereur Sévère, et pour subsister à la sueur de ma front, j'ai construit ces choses, passetemps pour les vivants. Et vous, amis, amusez-vous à entrer dans le labyrinthe tour à tour. Sauve le peuple des marbriers, Sarapis. Le lieu est le labyrinthe » (trad. L. Bricault).
} 


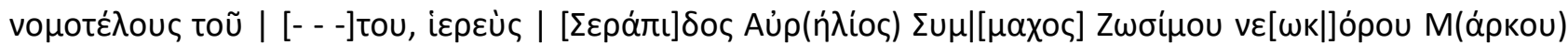

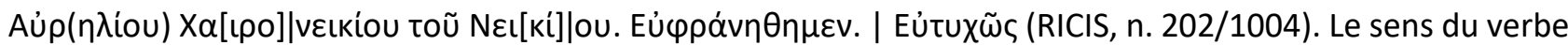
ne fait pas problème : « Nous avons banqueté dans une cérémonie rituelle ».

Les sources littéraires et les sources papyrologiques parlent de cette cérémonie religieuse. Des papyrus qui sont de vrais cartons d'invitation pour prendre part à ces banquets en honneur de Sarapis proviennent d'Oxyrhynque. II s'agit de fêtes organisées pour remercier Sarapis et une fois, c'est le dieu lui-

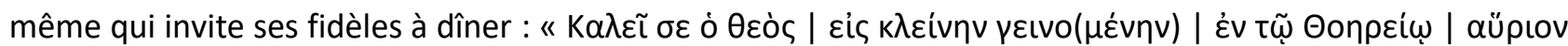

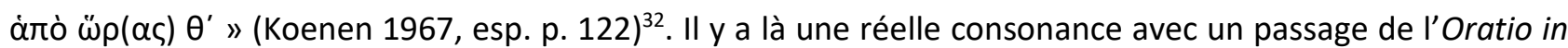
Sarapidem déjà citée:

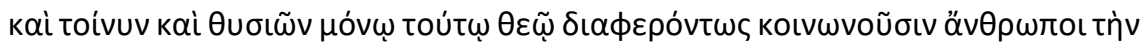

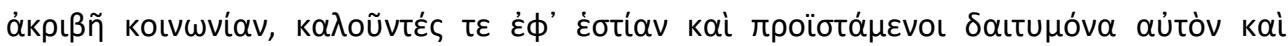

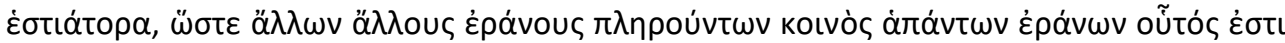

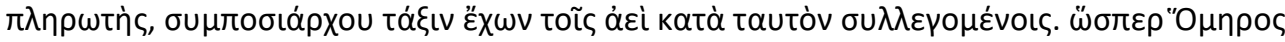

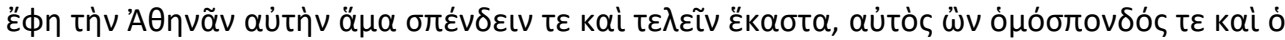

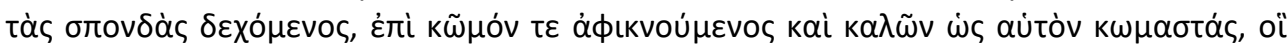

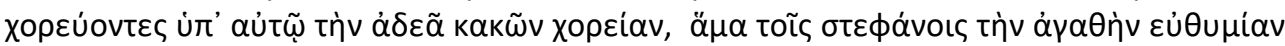

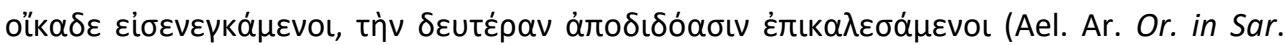
$27)^{33}$.

Le faste de ces banquets est rappelé par Tertullien. Comparant ces klinai avec le triclinium Christianorum, il écrit : Ad fumum cenae Serapiacae sparteoli excitabuntur (Tert. Apol. 39, 15) ${ }^{34}$. L'hagiographie fait aussi mention de ces banquets. Dans le récit conservé en grec et en copte de la conversion et du martyre des saints égyptiens Philémon et Apollonius, Satrius Arrien, gouverneur de la Thébaïde, invite Philémon à un banquet de Sarapis.

Voici le passage en grec :

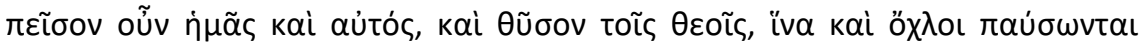

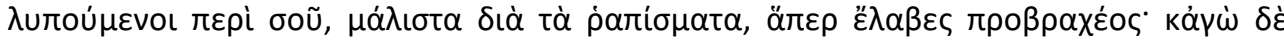

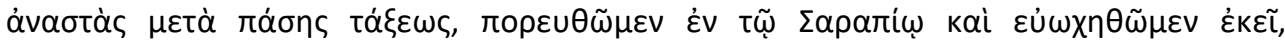

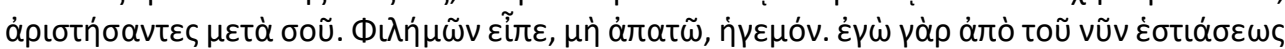

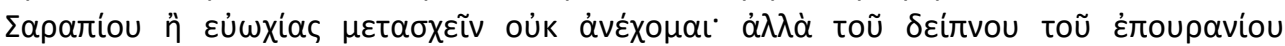

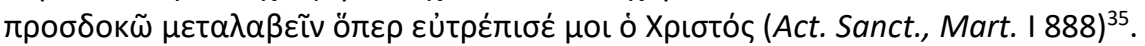

\footnotetext{
32 " Le dieu t'invite au banquet qui se tiendra dans le Thoerios, demain, à partir de la neuvième heure ".

${ }^{33}$ " Or ce dieu est aussi le seul que les hommes, avec un soin tout particulier, associent aux sacrifices, en l'invitant à leur table et en le mettant à leur tête en tant que convive et donneur de banquet. Par conséquent, si les autres contribuent chacun à des repas différents, celui-ci apporte sa contribution commune à tous les repas sans exception, en qualité de président du banquet pour ceux qui en toute occasion se réunissent en son nom. De même qu'Homère disait qu'Athéna elle-même à la fois fait des libations et exauce chaque vœu, il verse lui-même des libations et il en est le destinataire, il se rend à la fête et invite chez lui des festoyeurs qui, exécutant sous son autorité la danse sans mauvaises conséquences, après avoir apporté à la maison leur bonne humeur en même temps que leurs couronnes, lui rendent la pareille quand ils l'ont invité » (trad. Goeken).

34 «À cause de la fumée d'un banquet en l'honneur de Sarapis, on n'hésitera pas à appeler les pompiers ! »

35 " "Écoute-nous donc et sacrifie toi aussi aux dieux, de sorte que la foule puisse s'arrêter de pleurer pour toi, surtout à cause des coups dont tu as souffert il y a peu de temps. Me levant avec toute ma cour, allons au temple de Sarapis et
} 
Et en copte :

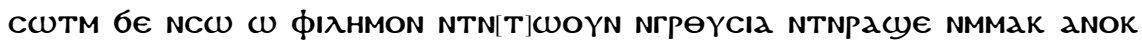
MN TTAżlC THPC. NTNTWOYN NTNBCK ETCIOOYN NTN.XWKM 21 OYCOT MNN[Ca NaI]

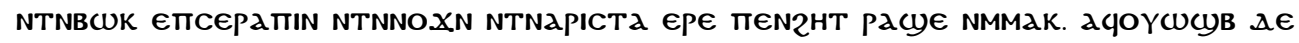

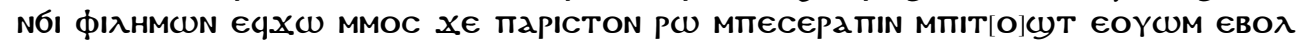

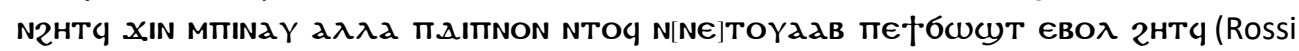
1893, p. 74$)^{36}$.

Le récit hagiographique grec est daté du $V^{\mathrm{e}} \mathrm{s}$. Il paraît évident, dès lors, que le souvenir des banquets de Sarapis persiste, ce qui démontre l'importance qu'ils avaient eu au niveau cultuel.

Qu'est-ce que peut signifier alors le mot $\lambda \alpha \beta u ́ p ı v \theta o \varsigma$ qui paraît si important au marbrier de Tripolis ? L. Robert, qui à bien étudié l'inscription, met en relation quatre mots du texte : d'un côté $\lambda \alpha \beta u ́ p ı v \theta o \varsigma$ et

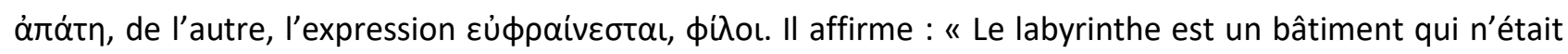
pas nécessairement de grandes dimensions, mais dont le plan était très compliqué... c'était un'árátn, un lieu

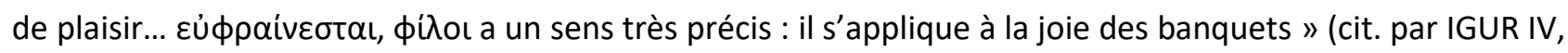
n. 1567).

banquetons richement en mangeant avec toi." Mais Philémon répondit: “Gouverneur, ne te fais pas d'illusion! Moi, à partir de ce moment, je ne supporterai plus de prendre part au banquet dans le temple de Sarapis, mais j'attends de participer au banquet céleste que le Christ m'a préparé!" "

${ }^{36}$ " "Écoute-moi, Philémon! Levons-nous, sacrifie, et moi et toute la cour, nous nous réjouirons avec toi. Levons-nous et allons aux bains, lavons-nous tous ensemble, puis nous irons chez Sarapis, nous nous mettrons à manger, notre cœur se réjouissant avec toi." Mais Philémon répondit en disant: "Le repas de Sarapis, dorénavant, j'ai décidé de ne plus le manger, mais c'est le dîner des saints que je vais contempler." " 


\section{ABRÉVIATIONS ET BIBLIOGRAPHIE}

BIANCHI, U. La religione greca, Torino : UTET, 1975.

CCID = HÖRIG, M., SCHWERTHEIM, E. (edd.), Corpus Cultus Iovis Dolicheni, Leiden - New York - København Köln: Brill, 1987.

DEPUYDT, L. (ed.). Encomiastica from the Pierpoint Morgan Library: Five Coptic Homilies Attributed to Anastatius of Euchaita, Epiphanius of Salamis, Isaac of Antinoe, Severian of Gabala, and Theopempus of Antioch, 2 voll., Lovanii: Brepols, 1993.

DERCHAIN, PH., HUBAUX, J. Vespasien au Sérapéum, Latomus, Bruxelles, Latomus, 12, p. 38-52, 1953.

DONADONI, S. Due testi oracolari copti, in GUARINO A., LA BRUNA L. (edd.). Synteleia V. Arangio Ruiz, Napoli : Jovene, 1964, p. 286-289 = Idem, Cultura dell'Antico Egitto. Scritti di Sergio F. Donadoni, Roma : Università di Roma "La Sapienza". Dipartimento di Scienze Storiche Archeologiche e Antropologiche dell'Antichità, 1989, p. 531-534.

IGUR = Moretti, L. (ed), Inscriptiones Graecae Urbis Romae, 4 voll., Romae : s.n., 1968-1990

KOENEN, L. Eine Einladung zur Kline des Sarapis. Zeischrift für Papyrologie und Epigraphik, Bonn : Habelt, 1, p. 121-126, 1967.

LIETZMANN, D.H. (ed.). Griechische Papyri², Berlin : Marcus - Weber, 1934.

MALAISE, M. Inventaire préliminaire des documents égyptiens découverts en Italie, Leiden: Brill 1972a

MALAISE, M. Les conditions de pénétration et de diffusion des cultes égyptiens en Italie, Leiden: Brill $1972 \mathrm{~b}$.

MORA, F. Prosopografia isiaca. I. Corpus prospographicum Religionis Isiacae. II. Prosopografia storica e statistica del culto isiaco, Leiden - New York - København - Köln: Brill, 1990.

PAPINI, L. Biglietti oracolari in copto dalla necropoli di Antinoe, en ORLANDI, T., WISSE, F. (edd.), Acts of the Second International Congress of Coptic Studies, Roma, 22-26 September 1980, Roma : C.I.M., 1985, p. 245-256.

PETERSON, E. EIS QEOS. Epigraphische, formgeschichtliche und religionsgeschichtliche Untersuchungen, Göttingen : Vandenhoeck \& Ruprecht, 1926.

PGM = Preisendanz, K (ed.). Papyri Magicae Graecae ${ }^{3}$, Stuttgardiae: Teubner, 1972-1974 (ed. or. 3 voll.; le tavole del III volume sembrerebbero essere andate distrutte [sic] nel 1944).

RICIS = Bricault, L. (ed.). Recueil des Inscriptions Concernant les Cultes Isiaques, 3 voll., Paris : De Boccard, 2005.

ROSSI, F. Un nuovo codice copto del Museo Egizio di Torino contenente la vita di s. Epifanio ed i martiri di s. Pantoleone, di Ascla, di Apollonio, di Filemone, di Ariano e di Dios con versetti di vari capitoli del Libro di Giobbe. Atti della Regia Accademia dei Lincei. V serie. Classe di Scienze morali, storiche e filologiche, Roma Bardi, , 1, p. 3-136, 1893.

SANZI, E. Mithras in the Magical Papyri. Religio-Historical Reflections on Various Magical Texts, en PACHIS, 


\section{ENNIO SANZI}

P., WIEBE, D. (edd.). Chasing Down Religion: In the Sights of History and the Cognitive Sciences, Essays in Honour of Luther H. Martin, Thessaloniki, 2010, p. 391-406 = Sheffield : Equinox, 2014, pp. 391-406. VIDMAN, L. Sylloge inscriptionum religionis Isiacae et Sarapiacae, Berlin : De Gruyter, 1969. 